Marquette University

e-Publications@Marquette

$5-1-2002$

Calculation of Damping Rates in Thin Inhomogeneous Ferromagnetic Films Due to Coupling to Lattice Vibrations

R. D. McMichael

National Institute of Standards \& Technology

Andrew Kunz

Marquette University, andrew.kunz@marquette.edu

Published version. Journal of Applied Physics, Vol. 91, No. 10 (May 2002). DOI. (C) 2002 American Institute of Physics. Used with permission. 


\title{
Calculation of damping rates in thin inhomogeneous ferromagnetic films due to coupling to lattice vibrations
}

\author{
R. D. McMichael ${ }^{\text {a) }}$ and Andrew Kunz \\ National Institute of Standards and Technology, Gaithersburg, Maryland 20899
}

\begin{abstract}
This article describes calculations of ferromagnetic resonance damping rates due to coupling between the magnetization and lattice vibrations through inhomogeneities. The mechanisms we have explored include generation of shear phonons through inhomogeneous anisotropy and generation of both longitudinal and shear phonons through inhomogeneous magnetostriction. In both cases, inhomogeneities couple the uniform precession to finite wave vector phonons. For both coupling mechanisms, the predicted damping rate is on the order of $10^{6} \mathrm{~s}^{-1}$ in transition metals. The damping rate by these mechanisms is inversely proportional to the fifth power of the shear phonon velocity, and may play a significant role in mechanically softer materials such as magnet/polymer nanocomposites. [DOI: 10.1063/1.1450831]
\end{abstract}

\section{INTRODUCTION}

It is well known that inhomogeneities can cause uniform precession modes to scatter into finite-wave vector spinwave excitations in a process described by the two-magnon model. ${ }^{1-7}$ The two-magnon process produces an effective line width in ferromagnetic resonance experiments, but it may be regarded as something other than true damping because the energy stays within the magnetization without being transferred to the lattice. The analogous process was illustrated in micromagnetic models of switching without damping. ${ }^{8}$

The inhomogeneities that allow the uniform, $\mathbf{k}=0$, precession mode to drive nonuniform precession modes with wave vector $\mathbf{k} \neq 0$ will also allow uniform precession to couple to $\mathbf{k} \neq 0$ sound waves in the film and substrate. The transfer of energy to sound waves would be a true damping effect, but its measurement by ferromagnetic resonance would be masked by the presence of two-magnon broadening. The purpose of this article is to estimate theoretically whether the inhomogeneities that enable two-magnon processes within the magnetic subsystem also enable a significant amount of true damping to sound waves in the lattice.

We consider a Hamiltonian that includes the uniform mode of precession, a manifold of phonons and interaction terms of the form

$$
\begin{aligned}
\frac{\mathcal{H}}{\hbar}= & \omega_{0} a^{\dagger} a+\sum_{\mathbf{k}, s} \omega_{s}(\mathbf{k}) c_{\mathbf{k}, s}^{\dagger} c_{\mathbf{k}, s}+\sum_{\mathbf{k}, s}\left(F_{\mathbf{k}, s} a c_{\mathbf{k}, s}^{\dagger}\right. \\
& \left.+F_{\mathbf{k}, s}^{*} a^{\dagger} c_{\mathbf{k}, s}\right),
\end{aligned}
$$

where $a, a^{\dagger}$ and $c_{\mathbf{k}, s}, c_{\mathbf{k}, s}^{\dagger}$ are the lowering and raising operators for the uniform precession mode and for phonons, respectively. Phonon modes are identified by wave vector $\mathbf{k}$, and polarization index $s$.

The decay rate for the uniform precession, $\Gamma$, is given by the golden rule of time dependent perturbation theory,

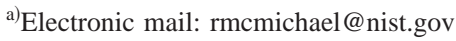

$$
\Gamma=\frac{2 \pi}{\hbar} \sum_{\mathbf{k}, s}\left|F_{\mathbf{k}, s}\right|^{2} \delta\left[\hbar \omega_{0}-\hbar \omega_{\mathrm{s}}(\mathbf{k})\right]
$$

To proceed with this formulation, we must determine the interaction coefficients $F_{\mathbf{k}, s}$ in terms of materials properties and the properties of the excitations.

For small deviations of the magnetization from the equilibrium direction, we write the magnetization as $\mathbf{M}=M_{\mathrm{s}} \hat{\rho}$ $+m_{\theta} \hat{\theta}+m_{\phi} \hat{\phi}$. The equilibrium magnetization direction is along the unit vector $\hat{\rho}$; $\hat{\theta}$ and $\hat{\phi}$ are chosen to lie along the principal axes of the uniform precession (see Fig. 1).

We write the energy of the magnetization in a uniform film as

$$
E=\mu_{0} V_{f}\left(\frac{1}{2} h_{\theta \theta} m_{\theta}^{2}+\frac{1}{2} h_{\phi \phi} m_{\phi}^{2}\right),
$$

where $h_{\theta \theta}$ and $h_{\phi \phi}$ are normalized fields and $V_{f}$ is the volume of the magnetic film. For magnetization aligned with an applied field $H_{a}$ in the plane of a film, $h_{\theta \theta}=H_{a} / M_{s}$ and $h_{\phi \phi}=\left(H_{a}+M_{s}\right) / M_{s}$.

In terms of $a$ and $a^{\dagger}, m_{\theta}$ and $m_{\phi}$ are given by

$$
m_{\theta, 0}=\frac{1}{i} \sqrt{\frac{\hbar \omega_{0}}{2 \mu_{0} V_{f} h_{\theta \theta}}}\left(a-a^{\dagger}\right),
$$

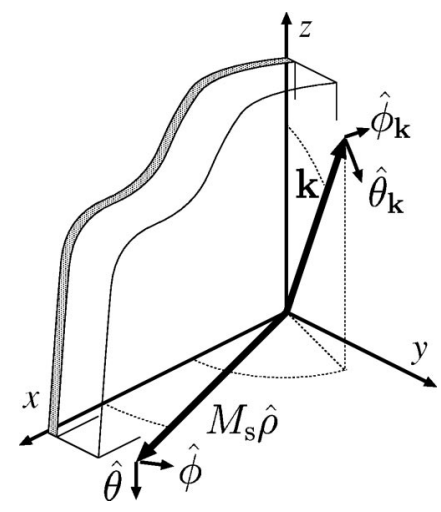

FIG. 1. Coordinate system used for the calculations. The film and substrate lie in the $x-z$ plane. Unit vectors $\hat{\theta}_{\mathbf{k}}$ and $\hat{\phi}_{\mathbf{k}}$ are chosen as polarization vectors for shear phonons. 


$$
m_{\phi, 0}=\sqrt{\frac{\hbar \omega_{0}}{2 \mu_{0} V_{f} h_{\phi \phi}}}\left(a+a^{\dagger}\right) .
$$

The displacement of atoms in the film and substrate can be written as ${ }^{9}$

$$
\mathbf{u}(\mathbf{r})=\sum_{\mathbf{k}, s} \sqrt{\frac{\hbar}{2 \rho V_{s} \omega_{s}(\mathbf{k})}}\left(c_{\mathbf{k} s}+c_{-\mathbf{k} s}^{\dagger}\right) \hat{\epsilon}_{s}(\mathbf{k}) e^{i \mathbf{k} \cdot \mathbf{r}},
$$

where $\rho$ is the substrate density, $V_{s}$ is the substrate volume, and $\omega_{\mathrm{s}}(\mathbf{k})$ is the angular frequency of a phonon with wave vector $\mathbf{k}$ and polarization vector $\hat{\boldsymbol{\epsilon}}_{s}$.

In Sec. II, we calculate the damping rate with the anisotropy as the spin lattice coupling, and in Sec. III, we calculate the direct-to-lattice damping rate due to magnetostriction.

\section{COUPLING VIA ANISOTROPY}

The phonon-generating process can be described classically. Imagine a small grain of anisotropic material in a uniform host. The precessing magnetization and the lattice in this grain will experience equal but opposite, time-dependent torques due to the local anisotropy. The relaxation of the lattice in response to these torques leads to the dissipation of magnetic energy by this mechanism.

For a material with an inhomogeneous anisotropy, the local energy density can be expanded in a Taylor series in $m_{\theta}$ and $m_{\phi}$. The first order terms give rise to magnetization ripple $^{10}$ and the second order terms are

$$
\begin{aligned}
E_{p}= & \frac{\mu_{0} V_{\mathrm{f}}}{N} \sum_{\mathbf{r}}\left[\frac{1}{2} h_{\theta \theta}(\mathbf{r}) m_{\theta}(\mathbf{r})^{2}+h_{\theta \phi}(\mathbf{r}) m_{\theta}(\mathbf{r}) m_{\phi}(\mathbf{r})\right. \\
& \left.+\frac{1}{2} h_{\phi \phi}(\mathbf{r}) m_{\phi}(\mathbf{r})^{2}\right] .
\end{aligned}
$$

Written in terms of Fourier coefficients

$$
\begin{aligned}
E_{p}= & \mu_{0} V_{f} \sum_{\mathbf{k}}\left[f_{\theta \theta,-\mathbf{k}} m_{\theta, \mathbf{k}} m_{\theta, 0}+f_{\phi \phi,-\mathbf{k}} m_{\phi, \mathbf{k}} m_{\phi, 0}\right. \\
& \left.+f_{\theta \phi,-\mathbf{k}}\left(m_{\theta, \mathbf{k}} m_{\phi, 0}+m_{\phi, \mathbf{k}} m_{\theta, 0}\right)\right],
\end{aligned}
$$

where only those terms involving the uniform precession are kept and the uniform and inhomogeneous parts of $h_{i j}(\mathbf{r})$ are separated

$$
h_{i j}(\mathbf{r})=h_{i j}+\sum_{\mathbf{k} \neq 0} f_{i j, \mathbf{k}} e^{i \mathbf{k} \cdot \mathbf{r}} .
$$

In Eq. (7), and in what follows, $m_{\theta}$ and $m_{\phi}$ are small magnetization changes due to rotations of $\mathbf{M}$ relative to the lattice. The magnetization with respect to the direction of the local crystal axes becomes $\mathbf{m}=\mathbf{m}_{0}+\mathbf{m}_{p}$, where $\mathbf{m}_{0}$ is the magnetization due to uniform precession magnons only and $\mathbf{m}_{p}$ is the effective magnetization due to local rotation of the lattice which is described by the rotation vector $\nabla \times \mathbf{u}$ :

$$
\begin{aligned}
\mathbf{m}_{p, \mathbf{k}} & =\frac{1}{N} \sum_{\mathbf{r}} M_{s} \hat{\rho}(\nabla \times u) e^{-i \mathbf{k} \cdot \mathbf{r}} \\
& =i \sqrt{\frac{\hbar}{2 \rho V_{s} \omega_{s}(\mathbf{k})}}\left(c_{\mathbf{k} s}+c_{-\mathbf{k} s}^{\dagger}\right)\left\{\mathbf{M}\left[\mathbf{k} \times \hat{\boldsymbol{\epsilon}}_{s}(\mathbf{k})\right]\right\} .
\end{aligned}
$$

Assigning the polarization vectors $\hat{\epsilon}_{1}$ and $\hat{\epsilon}_{2}$ to $\hat{\theta}_{\mathbf{k}}$ and $\hat{\phi}_{\mathbf{k}}$, respectively, the double cross product in Eq. (10) can be written

$$
\begin{aligned}
\mathbf{M} \times \mathbf{k} \times \hat{\theta}_{\mathbf{k}}= & -M_{s} k \cos \left(\phi_{\mathbf{k}}-\phi\right) \hat{\theta}, \\
\mathbf{M} \times \mathbf{k} \times \hat{\phi}_{\mathbf{k}}= & -M_{s} k\left[\cos \left(\theta_{\mathbf{k}}\right) \sin \left(\phi-\phi_{\mathbf{k}}\right) \hat{\theta}\right. \\
& \left.+\sin \left(\theta_{\mathbf{k}}\right) \hat{\phi}\right] .
\end{aligned}
$$

The perturbation energy [Eq. (8)] can now be expanded in terms of raising and lowering operators for magnons and phonons. Using Eqs. (11) and (12), the $\theta$ and $\phi$ components of $\mathbf{m}_{p}$ [Eq. (10)] can be identified and substituted for $m_{\theta, \mathbf{k}}$ and $m_{\phi, \mathbf{k}}$ and Eqs. (4) and (5) can be substituted for $m_{\theta, 0}$ and $m_{\phi, 0}$. For each polarization index $s$, the coefficients $F_{\mathbf{k}, s}$ of terms containing $c_{\mathbf{k}}^{\dagger} a$ can be identified by comparison with Eq. (1). The result is

$$
\begin{aligned}
\sum_{s}\left|F_{\mathbf{k}, s}\right|^{2}= & \mu_{0} V_{s} \frac{\left(\hbar k \gamma \mu_{0} M_{s}^{2}\right)^{2}}{4 \rho V_{f} \omega_{k} \omega_{0}} \\
& \times\left\{\left[\left|f_{\theta \theta, \mathbf{k}}\right|^{2} h_{\phi \phi}+\left|f_{\theta \phi, \mathbf{k}}\right|^{2} h_{\theta \theta}\right]\right. \\
& \times\left[\cos ^{2}\left(\phi-\phi_{\mathbf{k}_{r m}}\right)+\cos ^{2} \theta_{\mathbf{k}_{r m}} \sin ^{2}\left(\phi-\phi_{\mathbf{k}_{r m}}\right)\right] \\
& \left.+\left[\left|f_{\phi \phi, \mathbf{k}}\right|^{2} h_{\theta \theta}+\left|f_{\theta \phi, \mathbf{k}}\right|^{2} h_{\phi \phi}\right] \sin ^{2} \theta_{\mathbf{k}_{r m}}\right\} .
\end{aligned}
$$

In this expression we have assumed that the material has symmetry such that $\left\langle f_{i j}(\mathbf{r}) f_{k l}\left(\mathbf{r}+\mathbf{r}^{\prime}\right)\right\rangle=\left\langle f_{i j}(\mathbf{r}) f_{k l}\left(\mathbf{r}-\mathbf{r}^{\prime}\right)\right\rangle$ where $\langle f\rangle$ is the spatial average of $f$ and $i, j, k$, and $l$ stand for $\theta$ and $\phi$. With this assumption, $f_{i j, \mathbf{k}} f_{k l, \mathbf{k}}^{*}$ is real. Terms odd in $\phi-\phi_{\mathbf{k}_{r m}}$ have also been dropped because they will cancel in the sum over $\mathbf{k}$.

To proceed further, the properties of $\left|f_{i j, \mathbf{k}}\right|^{2}$ must be determined. As an example, we assume

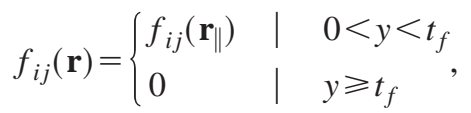

where $\mathbf{r}_{\|}$is a vector in the plane of the film, and we assume that $f_{i j}\left(\mathbf{r}_{\|}\right)$is correlated over a distance $D$ such that

$$
\left\langle f_{i j}\left(\mathbf{r}_{\|}\right) f_{i j}\left(\mathbf{r}_{\|}+\mathbf{r}_{\|}^{\prime}\right)\right\rangle=\left\langle f_{i j}^{2}\left(\mathbf{r}_{\|}\right)\right\rangle e^{-\left|\mathbf{r}_{\|}^{\prime}\right| / D} .
$$

Under these assumptions

$$
\left|f_{i j, \mathrm{k}}\right|^{2}=\frac{V_{f}}{V_{s}^{2}}\left(\frac{\sin \left(k_{y} t_{f} / 2\right)}{k_{y} t_{f} / 2}\right)^{2} \frac{2 \pi D^{2} t_{f}\left\langle f_{i j}^{2}\left(\mathbf{r}_{\|}\right)\right\rangle}{\left[1+\left(k_{\|} D\right)^{2}\right]^{3 / 2}},
$$

where $k_{\|}$is the in-plane component of $\mathbf{k}$.

To calculate $\Gamma$, the sum over $\mathbf{k}$ in Eq. (2) is converted to an integral, $\Sigma_{\mathbf{k}} \rightarrow V_{s} /(2 \pi)^{3} \int d \mathbf{k}$ for proper evaluation of the delta function. We assume a linear, isotropic dispersion relation for the shear phonons, $\omega_{\mathbf{k}}=c|\mathbf{k}|$ :

$$
\begin{aligned}
\Gamma= & \frac{1}{2 \pi} \int d \cos \theta_{\mathbf{k}} d \phi_{\mathbf{k}} d k \frac{\mu_{0} M_{s}^{2}\left(\gamma \mu_{0} M_{s}\right)^{2} \omega_{0}^{2}}{4 \rho c^{5}}\left[\frac{\sin \left(k_{y} t_{f} / 2\right)}{k_{y} t_{f} / 2}\right]^{2} \\
& \times \frac{D^{2} t_{f}}{\left[1+\left(k_{\|} D\right)^{2}\right]^{3 / 2}} \delta\left(|\mathbf{k}|-\omega_{0} / c\right)\left\{\left[\left\langle f_{\theta \theta}^{2}\right\rangle h_{\phi \phi}+\left\langle f_{\theta \phi}^{2}\right\rangle h_{\theta \theta}\right]\right. \\
& \times\left[\cos ^{2}\left(\phi-\phi_{\mathbf{k}_{r m}}\right)+\cos ^{2} \theta_{\mathbf{k}_{r m}} \sin ^{2}\left(\phi-\phi_{\mathbf{k}_{r m}}\right)\right] \\
& \left.+\left[\left\langle f_{\phi \phi}^{2}\right\rangle h_{\theta \theta}+\left\langle f_{\theta \phi}^{2}\right\rangle h_{\phi \phi}\right] \sin ^{2} \theta_{\mathbf{k}_{r m}}\right\} .
\end{aligned}
$$


For high frequencies, when the delta function in Eq. (1) selects large values of $k$ such that $|\mathbf{k}| t_{f} \gg 1$ and $|\mathbf{k}| D \gg 1$. The terms on the second line of Eq. (17) decrease with $k$, so the damping rate will decrease like $1 / \omega^{3}$ for large $\omega$.

For small $\mathbf{k}$ such that $|\mathbf{k}| t_{f} \ll 1$ and $|\mathbf{k}| D \ll 1$, the terms on the second line of Eq. (17) are approximately $D^{2} t_{f}$, and the damping rate can be approximated by

$$
\begin{aligned}
\Gamma \approx & \frac{\mu_{0} M_{s}^{2} D^{2} t_{f}\left(\gamma \mu_{0} M_{s}\right)^{2} \omega^{2}}{8 \pi \rho c^{5}}\left[h_{\theta \theta}\left(\left\langle f_{\phi \phi}^{2}\right\rangle+\left\langle f_{\theta \phi}^{2}\right\rangle\right)\right. \\
& \left.+h_{\phi \phi}\left(\left\langle f_{\theta \theta}^{2}\right\rangle+\left\langle f_{\theta \phi}^{2}\right\rangle\right)\right] .
\end{aligned}
$$

For low $\omega$, the damping rate is proportional to the square of the frequency. We expect maximum damping when the correlation length and/or film thickness are on the order of a phonon wavelength.

Note that the damping rate depends sensitively on a number extrinsic materials parameters including the correlation length $D$, and the thickness of the film. If we use orderof-magnitude magnetic parameters typical of Permalloy films, $\left(M_{s}=8 \times 10^{5} \mathrm{~A} / \mathrm{m}\right.$, and $\left.D=t_{f}=50 \mathrm{~nm}\right)$ mechanical properties typical of silicon, $\left(\rho=2.3 \times 10^{3} \mathrm{~kg} / \mathrm{m}^{3}, c=3\right.$ $\left.\times 10^{3} \mathrm{~m} / \mathrm{s}\right)$ and $\omega=2 \pi \times 10 \mathrm{GHz}$, and if we allow for a very large perturbation field on the same order as $M_{s}$, such that $\left\langle f_{i j}^{2}\right\rangle=1$, the damping rate due to generation of phonons is still only on the order of $10^{6} \mathrm{~s}^{-1}$, much smaller than rates of $10^{9} \mathrm{~s}^{-1}$ that are typically observed in ferromagnetic resonance experiments. Note that Eq. (17) depends on the speed of sound for transverse waves as $c^{-5}$. For a magnet/polymer composite with a lower speed of sound, the damping by this mechanism may be large enough to be dominant.

\section{COUPLING VIA MAGNETOSTRICTION}

Magnetostrictive coupling between magnetization precession modes and phonons have been observed as fine structure in the ferromagnetic resonance of the garnet due to standing sound waves in the substrates. ${ }^{11-13}$ Abrahams and Kittel $^{14}$ calculated the damping rate in single crystals due to a process where magnon scattering is accompanied by emission or absorption of a phonon. Suhl ${ }^{15}$ has calculated damping by generation of shear sound waves in particles much smaller than the wavelength of sound and viscous behavior of the lattice. In both of these works, the damping rate is small compared to experimental values.

We outline a calculation of the damping rate due to magnetostrictive generation of phonons in polycrystalline films using the the cubic magnetoelastic coupling energy given by

$$
\begin{aligned}
E_{\text {magel }}= & \frac{V_{f}}{N} \sum_{\mathbf{r}}\left[B_{1}\left(e_{x x} \alpha_{1}^{2}+e_{y y} \alpha_{2}^{2}+e_{z z} \alpha_{3}^{2}\right)\right. \\
& \left.+B_{2}\left(e_{x y} \alpha_{1} \alpha_{2}+e_{y z} \alpha_{2} \alpha_{3}+e_{z x} \alpha_{3} \alpha_{1}\right)\right],
\end{aligned}
$$

where $e_{i j}$ is the strain tensor and $\alpha_{i}$ is a direction cosine of the magnetization relative to the local crystallographic axes; $\alpha_{i}=m_{i} / M_{s}$. The strain is given by

$$
e_{i j}=\frac{1}{2}\left(\frac{\partial u_{i}}{\partial x_{j}}+\frac{\partial u_{j}}{\partial x_{i}}\right)
$$

Because the directions of the crystallographic axes vary from grain to grain in the film, we define a unitary coordinate transformation matrix $\mathbf{U}(\mathbf{r})$ such that $\alpha_{i}=\Sigma_{j} U_{i j}(\mathbf{r}) M_{j} / M_{s}$. The next step in the calculation would be to use Eqs. (4), (5), and (6) to expand Eq. (19) in terms of magnon and phonon raising and lowering operators. Because $\alpha_{i}$ is a linear combination of $M_{s}, m_{\theta}$, and $m_{\phi}$, products $\alpha_{i} \alpha_{j}$ will contain cross terms proportional to $m_{\theta} / M_{s}$ and $m_{\phi} / M_{s}$ with coefficients on the order of unity that depend on $\mathbf{r}$. With these substitutions, the coupling energy takes a form similar to Eq. (8) with terms $B g_{-\mathbf{k}} /\left(\mu_{0} M_{s}^{2}\right)$ in place of $f_{i j,-\mathbf{k}}$ where $g_{-\mathbf{k}}$ is a Fourier component of coefficients of the $\mathbf{r}$-dependent coordinate transformation from local crystallographic axes to the lab coordinates.

For transition metals with magnetostriction on the order of $10^{-5}, B$ is typically of $10^{6} \mathrm{~J} / \mathrm{m}$, the same order of magnitude as $\mu_{0} M_{s}^{2}$. Based on this estimate, damping to phonons via magnetostriction and damping to phonons via magnetocrystalline anisotropy are similar in magnitude. Perhaps the similar damping rates predicted for these two mechanisms should not be surprising since both magnetostriction and magnetocrystalline anisotropy have their origins in spin-orbit coupling.

In conclusion, the results of these calculations indicate that inhomogeneous coupling between the magnetization and lattice vibrations may be strong enough to give measurable damping in transition metal thin films. For magnet/polymer nanocomposites or other materials with low sound speeds, damping by phonon generation may play a much more significant role.

\section{ACKNOWLEDGMENT}

The authors acknowledge the support of the NIST nanotechnology program.

${ }^{1}$ A. M. Clogston, H. Suhl, L. R. Walker, and P. W. Anderson, J. Phys. Chem. Solids 1, 129 (1956)

${ }^{2}$ M. Sparks, R. Loudon, and C. Kittel, Phys. Rev. 122, 791 (1961).

${ }^{3}$ H. B. Callen, Fluctuation, Relaxation and Resonance in Magnetic Systems (Oliver and Boyd Ltd., Edinburgh, 1962), Chap. 4, pp. 69-85.

${ }^{4}$ C. W. Hass and H. B. Callen, in Magnetism, edited by G. T. Rado and H. Suhl (Academic, New York, 1963), Chap. 10, pp. 480-497.

${ }^{5}$ M. J. Hurben and C. E. Patton, J. Appl. Phys. 83, 4344 (1998).

${ }^{6}$ R. D. McMichael, M. D. Stiles, P. J. Chen, and W. F. Egelhoff, Jr., J. Appl. Phys. 83, 7037 (1998)

${ }^{7}$ R. Arias and D. L. Mills, Phys. Rev. B 60, 7395 (1999).

${ }^{8}$ V. L. Safonov and H. N. Bertram, Phys. Rev. B 63, 094419 (2001).

${ }^{9}$ N. W. Ashcroft and N. D. Mermin, Solid State Physics (Saunders College, West Washington Square, Philadelphia, PA 19105, 1976), Apppendix L.

${ }^{10}$ K. J. Harte, J. Appl. Phys. 39, 1503 (1968).

${ }^{11}$ H. Dötch, Appl. Phys. 15, 167 (1978).

${ }^{12}$ R. J. Yeh, P. E. Wigen, and H. Dötch, Solid State Commun. 44, 1183 (1982)

${ }^{13}$ D. A. Romanov and E. G. Rudashevsky, IEEE Trans. Magn. 29, 3405 (1993).

${ }^{14}$ E. Abrahams and C. Kittel, Phys. Rev. 88, 1200 (1952).

${ }^{15}$ H. Suhl, IEEE Trans. Magn. 34, 1834 (1998). 